\title{
¿Aritmética elemental o Geometría elemental? Un estudio en futuros maestros
}

\section{Arithmetic elementary or Geometry elementary? A study about prospective teachers}

\author{
Rosa Nortes Martínez-Artero ${ }^{1}$ \\ mrosa.nortes@um.es \\ Andrés Nortes ChecA \\ anortes@um.es \\ Universidad de Murcia, España
}

\begin{abstract}
Resumen:
Las pruebas de conocimientos matemáticos elementales en el Grado de Maestro de Primaria son necesarias al inicio de las asignaturas de Matemáticas y su didáctica. Se ha elegido la Prueba de Conocimientos y Destrezas Indispensables (CDI) para los alumnos de $6 .^{\circ}$ de Primaria de la Comunidad de Madrid y se han seleccionado los problemas de Aritmética y Geometría de las pruebas de 2011 a 2014 aplicándolas a los futuros maestros en los cursos 11/12 al 14/15. De los 390 alumnos que constituyen la muestra en dos cursos los alumnos obtienen resultados positivos por encima del $50 \%$ en Aritmética elemental, y el mayor porcentaje de aciertos en Geometría elemental es del $38 \%$, lo que nos indica que las matemáticas elementales no las tienen aprendidas y si lo fueron, las tienen olvidadas.
\end{abstract}

Palabras clave:

Pruebas; aritmética elemental; geometría elemental; maestros; primaria.

\begin{abstract}
:
Mathematical knowledge tests in the Primary Education Degree are necessary at the start of the subject Mathematics and its Didactics. The Indispensable Knowledge and Skills Test for students of 6th of Primary in the Region of Madrid has been chosen and the arithmetics and geometry problems from 2011 and 2014 selected, giving them to the degree students during academic years from $11 / 12$ to $14 / 15$. Out of the 390 subjects the sample consist of, in two of the years analyzed the students obtain marks above $50 \%$ in elementary arithmetics. The highest mark in elementary geometry is $38 \%$. This indicates that the students' elementary mathematics knowledge is poor or, given it was ever good, students have lost it.
\end{abstract}

\section{Key words:}

Tests; elementary arithmetics; elementary geometry; teachers; primary school education.

\section{Dirección para correspondencia (correspondence address):}

Rosa Nortes Martínez-Artero. Departamento de Didáctica de las Ciencias Matemáticas y Sociales. Facultad de Educación. Universidad de Murcia. Campus de Espinardo, s/n. 30100 Murcia (España) 


\section{Résumé:}

Les épreuves de connaissances mathématiques élémentaires dans le «Grado de Maestro de Primaria» sont nécessaires au début des matières de Mathématiques et sa didactique. Nous avons choisi la Prueba de Conocimientos y Destrezas Indispensables (CDI) /I'Epreuve de Connaissances et d'Adresses Indispensables (CAI) pour les élèves de sixième année de Primaire de la Communauté de Madrid et nous avons sélectionné les problèmes d'Arithmétique et de Géométrie des épreuves de 2011 à 2014 en les appliquant aux futurs professeurs des écoles lors des années 2011/2012 à 2014/2015. Des 390 élèves qui constituent l'échantillon des deux années, plus de $50 \%$ des élèves obtiennent des résultats positifs en Arithmétique élémentaire, et le pourcentage le plus haut en Géométrie élémentaire est de $38 \%$, ce qui nous indique que les mathématiques élémentaires ne sont pas apprises ou si elles l'ont été, sont oubliées.

\section{Mots clés:}

Épreuves; arithmétique élémentaire; géométrie élémentaire; professeurs des écoles; primaire.

Fecha de recepción: 26-12-2015

Fecha de aceptación: 10-2-2016

\section{Introducción}

Los futuros maestros acceden a los estudios del Grado con unos conocimientos matemáticos muy diversos que deben de incluir los correspondientes a Enseñanza Primaria.

El Real Decreto 126/2014 por el que se establece el currículo básico de la Educación Primaria establece como séptimo objetivo "Desarrollar las competencias matemáticas básicas e iniciarse en la resolución de problemas que requieran la realización de operaciones elementales de cálculo, conocimientos geométricos y estimaciones, así como ser capaces de aplicarlos a las situaciones de su vida cotidiana" (p. 19354). Y al referirse a Matemáticas indica:

En la Educación Primaria se busca alcanzar una eficaz alfabetización numérica, entendida como la capacidad para enfrentarse con éxito a situaciones en las que intervengan los números y sus relaciones, permitiendo obtener información efectiva, directamente o a través de la comparación, la estimación y el cálculo mental o escrito. (p. 19386).

En el Grado de Maestro de Educación Primaria, los contenidos de las asignaturas Matemáticas, Matemáticas y su didáctica o Didáctica de las Matemáticas, siguiendo la Orden ECI/385/2007, deben de conseguir las 
competencias en la materia Enseñanza y aprendizaje de las Matemáticas: adquirir competencias matemáticas básicas; conocer el currículo escolar de matemáticas; analizar, razonar y comunicar propuestas matemáticas; plantear y resolver problemas vinculados con la vida cotidiana, entre otras.

Las matemáticas elementales (Castro, Mengual, Prat, Albarracín, Gorgorió, 2014) son fundamentales, básicas, primarias, como conocimiento inicial para el aprendizaje de las Matemáticas. Y definen el conocimiento matemático fundamental para el Grado de Maestro Educación Primaria como "el conocimiento disciplinar en matemáticas necesario para seguir con aprovechamiento las materias de matemáticas y Didáctica de las Matemáticas" (p. 229).

El Profesor de Primaria debe tener unos conocimientos matemáticos suficientes, debe saber más de lo que tiene que enseñar y si el $91 \%$ de los alumnos encuestados por Ruiz de Gauna, García y Sarasua (2013) dice que las Matemáticas son fundamentales o bastante importantes, el futuro maestro debe de tener la preparación adecuada, siendo Aritmética y Geometría los pilares fundamentales de esas matemáticas elementales.

\section{Marco teórico}

El maestro de Primaria debe de saber aplicar los estándares de aprendizaje evaluables, que permiten definir los resultados de aprendizaje y concretan lo que el alumno debe saber, comprender y saber hacer en Matemáticas, debiendo ser observables, medibles y evaluables. En el RD 126/2014, tanto en los bloques de Números como de Geometría se establecen así:

1) Resuelve problemas que impliquen dominio de los contenidos trabajados, utilizando estrategias heurísticas, de razonamiento (clasificación, reconocimiento de las relaciones, uso de contraejemplos), creando conjeturas, construyendo, argumentando, y tomando decisiones, valorando las consecuencias de las mismas y la conveniencia de su utilización.

2) Reflexiona sobre el proceso aplicado a la resolución de problemas: revisando las operaciones utilizadas, las unidades de los resultados, comprobando e interpretando las soluciones en el contexto, buscando otras formas de resolverlo. (pp. 19390 y 19393). 
Castro, Gorgorió y Prat (2015) consideran que la Aritmética es una componente básica de las Matemáticas en la Escuela Primaria por lo que también debiera serlo en la formación matemática de los futuros maestros. Sin, embargo cuando Ruiz de Gauna et al. (2013) al preguntar a una muestra de alumnos, estudiantes del Grado de Maestro de Primaria que indicaran de entre los posibles integrantes del currículo de Primaria (álgebra, análisis, aritmética, estadística, geometría, historia de las matemáticas y recursos informáticos) cuáles eran los que entraban, el $91 \%$ considera la geometría, mientras que la aritmética solo el $40 \%$, quizás debido al desconocimiento del término aritmética que no fue asociado a números.

Torregrosa, Quesada y Penalva (2010) indican que trabajar con pruebas de geometría ayuda a pensar de manera lógica y a desarrollar argumentos coherentes que expliquen por qué un resultado es cierto. Por lo que las destrezas de los alumnos en la visualización y en el razonamiento sobre relaciones espaciales son fundamentales en geometría y en números deben adquirir fluidez de cálculo, entendida como la habilidad para calcular eficientemente y con exactitud con fracciones, decimales y enteros (NCTM, 2000).

Los estudiantes que inician los estudios del Grado de Maestro de Primaria deben dominar los contenidos referidos a las matemáticas escolares, en el sentido de recordar conocimientos adquiridos en las primeras etapas de la enseñanza (Salinas, 2007) y en el currículo de Matemáticas para Primaria en la Región de Murcia (Decreto 198/2014) se indica "el área de Matemáticas debe construir los fundamentos del razonamiento lógico en los niños y niñas de esta etapa" (p. 33184).

Montes, Contreras, Liñán, Muñoz-Catalán, Climent y Carrillo (2015) indican que es imprescindible que los futuros maestros partan de un conocimiento matemático, solidamente construido para así abordar con garantías de éxito el conocimiento didáctico del contenido y profundizar en el conocimiento matemático especializado que requiere un maestro para realizar una buena práctica docente.

Son muchos los autores que aseguran que el conocimiento que poseen los estudiantes para maestro está muy lejos de poder calificarse de idóneo o adecuado para el desempeño de su futura labor docente. (Escudero-Domínguez y Carrillo, 2014).

Nuestro propósito es conocer si las matemáticas elementales en Aritmética y Geometría constituyen un conocimiento que los estudiantes 
traen consigo o por el contrario constituyen un conocimiento matemático incompleto, porque la falta de conocimiento del contenido genera una dificultad a la hora de crear representaciones útiles en la enseñanza y no permite abordar con garantías las componentes del conocimiento didáctico del contenido. Liñan y Contreras (2013) se hacen eco de numerosos autores que señalan algunas de las deficiencias de los estudiantes para maestro en el ámbito de la aritmética en su formación y apuntan la necesidad de mejorar los procesos de selección de los candidatos a Maestros en cuanto a sus conocimientos matemáticos básicos. Y García, Buforn y Torregrosa (2014) nos recuerdan que los contenidos geométricos suelen ser olvidados o tratados superficialmente en los currículos de primaria y secundaria debido a un mayor énfasis en la aritmética, en la educación primaria y en el álgebra en la educación secundaria y como consecuencia, es normal que los estudiantes para maestro tengan un conocimiento limitado sobre los contenidos y procesos geométricos.

El presente trabajo trata de conocer si los alumnos que Ilegan al Grado de Maestro de Primaria saben resolver problemas elementales de Aritmética y Geometría y para ello se utiliza una prueba estandarizada de 6 . $^{\circ}$ de Primaria que se aplica cada año a más de cincuenta mil estudiantes de este nivel en la Comunidad Autónoma de Madrid.

\section{Método}

\section{Diseño}

Al inicio de cada curso académico, se pasa a los alumnos del Grado de Maestro de Primaria una prueba de conocimientos elementales antes del comienzo de las asignaturas de Matemáticas y su didáctica. Se toma la Prueba de Matemáticas CDI que aplican en la Comunidad de Madrid a los alumnos de $6 .^{\circ}$ de Primaria en el mes de mayo. De estas pruebas se seleccionan los problemas de Aritmética y Geometría.

\section{Muestra}

La muestra la componen 390 alumnos de $2 .^{\circ}$ curso del grado de Maestro de Primaria de la Universidad de Murcia, distribuidos así: 70 de un grupo del curso 2011/12, 189 de tres grupos del curso 2012/13, 71 alumnos 
de un grupo del curso 2013/14 y 60 alumnos de un grupo del curso 2014/2015, todos ellos elegidos por conveniencia por tener acceso a ellos. Se pasaron las pruebas un día determinado de principio de curso, contestando aquellos alumnos que se encontraban en clase.

\section{Instrumentos}

De las Pruebas de Conocimientos y Destrezas Indispensables (Pruebas CDI) que anualmente la Comunidad de Madrid (CAM, 2014) aplica a los alumnos de $6 .^{\circ}$ curso de Educación Primaria, se han seleccionado los problemas de Aritmética y de Geometría correspondientes a los años 2011 (uno de Aritmética y uno de Geometría), 2012 (uno de Aritmética y uno de Geometría), 2013 (uno de Aritmética y uno de Geometría) y 2014 (dos de Aritmética y dos de Geometría), que vienen recogidos en la tabla 1.

Tabla 1. Enunciados de problemas de Aritmética y Geometría.

\begin{tabular}{lc}
\hline \multicolumn{1}{c}{ ARITMÉTICA } & GEOMETRÍA \\
\hline 7. En el colegio de Pablo hay 500 & 8. El lado del cuadrado ABCD mide 2 \\
alumnos. Una quinta parte juega al & $\mathrm{cm}$. Tomando como lado su diagonal, \\
fútbol, el 30 \% juega al baloncesto y & BD, se construye un nuevo cuadrado, \\
el resto no practica ningún deporte. & DBEF, tal como se indica en la figura. \\
a) ¿Cuántos alumnos del colegio de & Calcula: \\
Pablo juegan al baloncesto? & \\
b) ¿Cuántos alumnos no hacen nin- \\
gún deporte?
\end{tabular}




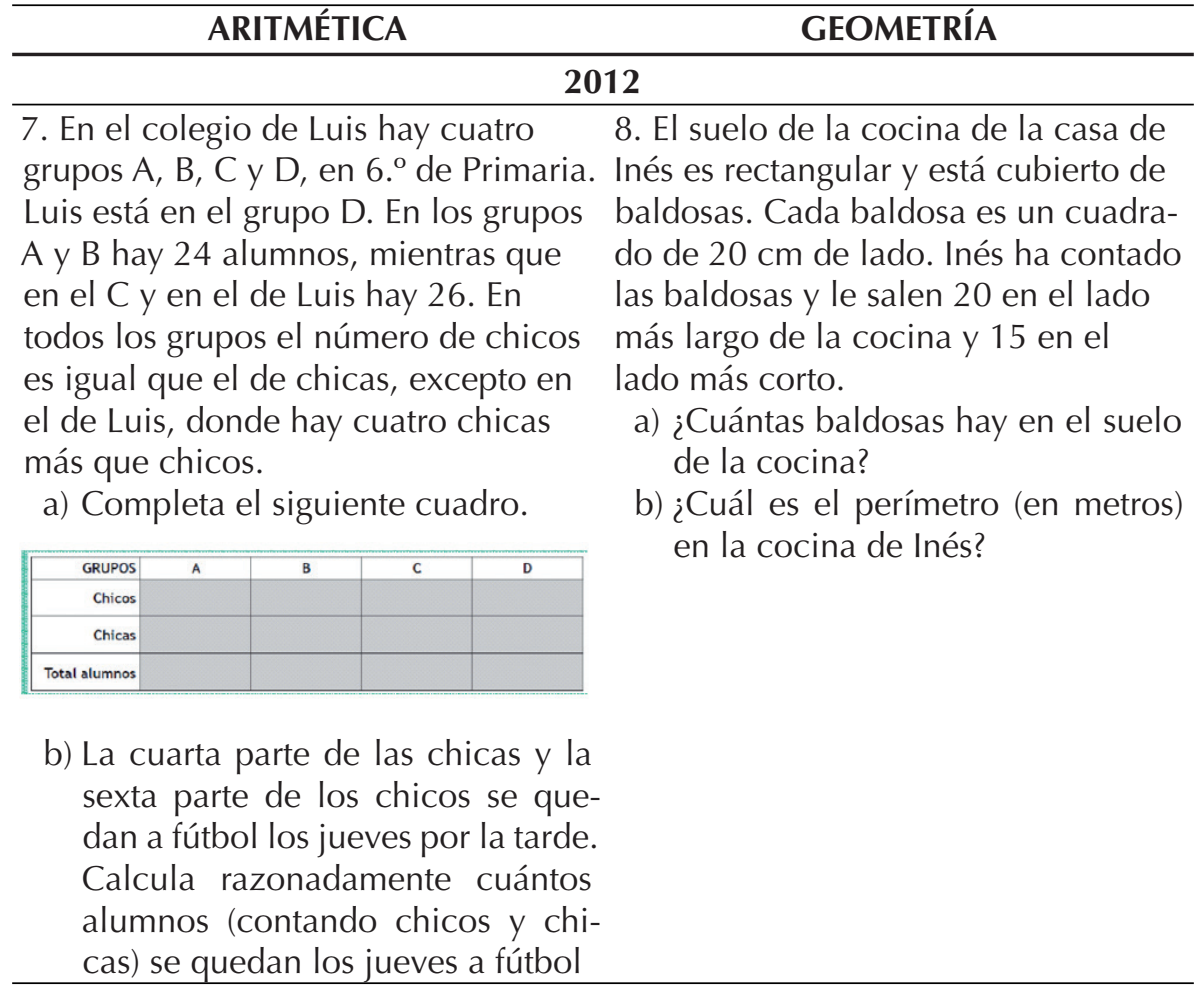

\section{3}

8. Jaime ha comprado un libro que le 7. En el colegio de Pedro hay un camha costado 16,50 euros, tres cuadernos de 3,25 euros cada uno y cuatro bolígrafos de 2,20 euros cada uno.

a) ¿Cuánto ha gastado Jaime en total? po de fútbol que mide $100 \mathrm{~m}$ de lado y $60 \mathrm{~m}$ de ancho. Por el interior del campo, a $5 \mathrm{~m}$ del borde, se ha traza-

b) Ha pagado con un billete de 50 euros. ¿Cuánto dinero le tienen que devolver?

do una línea tal y como está indicado

en la figura

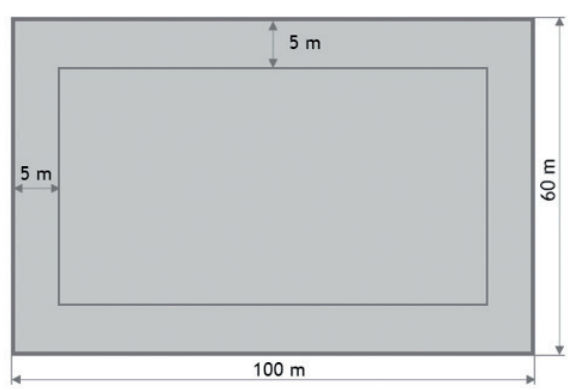

a) ¿Qué distancia recorre Pedro si da una vuelta al campo justo por el borde exterior? 


\begin{tabular}{cc}
\hline ARITMÉTICA & GEOMETRÍA \\
\hline b) ¿Qué distancia recorre Pedro si da \\
una vuelta siguiendo la línea tra- \\
zada por el interior del campo? \\
c) Calcula el área del campo de fút- \\
bol \\
\hline $\mathbf{2 0 1 4}$
\end{tabular}

5. En clase de $6 .{ }^{\circ} \mathrm{B}$ hay 30 alumnos. 9. Halla razonadamente el perímetro En la última evaluación de Matemáti- de la figura (los ángulos son todos cas, la quinta parte ha sacado sobre- rectos) saliente y el $20 \%$ ha suspendido.

a) ¿Cuántos alumnos han aprobado?

b) ¿Cuántos alumnos han sacado sobresaliente?

19

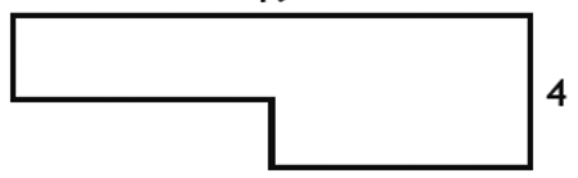

8. La profesora ha repartido una jarra con 2 litros de zumo de naranja entre 8 alumnos. A todos ellos les ha dado la misma cantidad de zumo.

a) ¿Cuántos litros ha dado a cada uno?

b) ¿Cuántos centilitros ha dado a cada uno?
10. El área de cada uno de los nueve cuadrados pequeños es 9 .

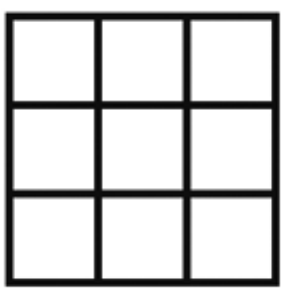

a) ¿Cuál es el perímetro del cuadrado grande?

b) ¿Cuál es el área del cuadrado grande?

\section{Resultados}

Los apartados de cada problema han sido puntuados con 0 o con 1 según estuvieran mal o bien. Si están bien todos los apartados el problema se puntúa con 1 y en caso contrario con 0 . Para el total se sigue el mismo procedimiento y cuando hay más de un problema, si todos están bien se puntúa con un 1 y en caso contrario con un 0 .

Señalamos con A7a el problema 7 de Aritmética, apartado a), con G8b el problema 8 de Geometría, apartado b), con A7 y con G8 los totales englobando los apartados de cada problema y con A y G los totales 
de los dos problemas en 2014, puntuándolos con 1 si todos los apartados tienen 1 y en otros casos, con 0.

\section{Resultados 2011 (curso 2011/12)}

La prueba de 2011 fue aplicada a los 70 alumnos presentes de un grupo de $2 .^{\circ}$ del Grado de Maestro de Primaria al inicio del curso 2011/12 y los resultados por apartado de cada problema y totales aparecen en la tabla 2.

Tabla 2. Resultados problemas Aritmética y Geometría 2011.

\begin{tabular}{ccccccc}
\hline $\mathbf{2 0 1 1 / 1 2}$ & A7a & A7b & A7 & G8a & G8b & G8 \\
\hline Media & 0,886 & 0,857 & 0,829 & 0,571 & 0,114 & 0,086 \\
D. T. & 0,320 & 0,352 & 0,380 & 0,498 & 0,320 & 0,282 \\
Casos & 70 & 70 & 70 & 70 & 70 & 70 \\
\hline
\end{tabular}

- El 88,6 \% responde bien el apartado a) de la cuestión 7 de Aritmética, el 85,7 \% el apartado b) y el 82,9\% los dos.

- El 57,1\% responde bien el apartado a) de la cuestión 8 de Geometría, el $11,4 \%$ el apartado b) y el 8,6 \% los dos.

- El número de alumnos que responde bien en Aritmética es casi diez veces el de Geometría.

Se recoge en la tabla 3 el número de alumnos que contestó bien y mal a los problemas de Aritmética y Geometría.

Tabla 3. Número de alumnos por respuesta 2011.

\begin{tabular}{cccc}
\hline A7/G8 & $\mathbf{0}$ & $\mathbf{1}$ & Total \\
\hline $\mathbf{0}$ & 12 & 0 & 12 \\
$\mathbf{1}$ & 52 & 6 & 58 \\
\hline Total & 64 & 6 & 70 \\
\hline
\end{tabular}

La correlación A7-G8 es $r=0,139(p=.250)$ y no es significativa.

- Solo 6 alumnos responden bien al problema de Aritmética y al de Geometría, que representan el 8,6\%.

- Hay 12 alumnos que no responden bien a ninguno de los dos problemas, el 17,1\%.

- La correlación entre A7 y G8, es baja y no significativa. 
Por género se aplica la t-Student para ver si existen diferencias significativas entre alumnos y alumnas. Las puntuaciones y probabilidad vienen en la tabla 4.

Tabla 4. Resultados 2011 por género.

\begin{tabular}{ccccccc}
\hline $\mathbf{2 0 1 1 / 1 2}$ & A7a & A7b & A7 & G8a & G8b & G8 \\
\hline Hombre & 0,909 & 0,864 & 0,864 & 0,545 & 0,136 & 0,091 \\
Mujer & 0,875 & 0,854 & 0,812 & 0,583 & 0,104 & 0,083 \\
$\boldsymbol{p}$-valor & .683 & .918 & .604 & .770 & .699 & .918 \\
\hline
\end{tabular}

- No hay diferencias significativas por género en cada uno de los apartados ni en el total.

- Donde los resultados son más próximos es en el problema completo de Geometría $(p=.918)$ y en el apartado b) de Aritmética ( $p$ $=.918)$.

\section{Resultados 2012 (curso 2012/13)}

La prueba de 2012 fue aplicada a los 189 alumnos de tres grupos de 2. del Grado de Maestro de Primaria al inicio del curso 2012/13, agrupando los resultados en la tabla 5.

Tabla 5. Resultados problemas Aritmética y Geometría 2012.

\begin{tabular}{ccccccc}
\hline 2012/13 & A7a & A7b & A7 & G8a & G8b & G8 \\
\hline Media & 0,439 & 0,280 & 0,270 & 0,725 & 0,302 & 0,265 \\
D. T. & 0,498 & 0,450 & 0,445 & 0,448 & 0,460 & 0,454 \\
Casos & 189 & 189 & 189 & 189 & 189 & 189 \\
\hline
\end{tabular}

- El 43,9\% responde bien al apartado a) de la cuestión 7 de Aritmética, el $28 \%$ al apartado b) y el $27 \%$ a los dos.

- El 72,5\% responde bien al apartado a) de la cuestión 8 de Geometría, el 30,2 \% al apartado b) y el 26,5\% a los dos.

- El número de alumnos que responde bien en Aritmética es aproximadamente el mismo que responde bien en Geometría.

El número de alumnos que contestó bien y mal a cada problema se recoge en la tabla 6 de doble entrada. 
Tabla 6. Número de alumnos por respuesta 2012.

\begin{tabular}{cccc}
\hline A7/G8 & $\mathbf{0}$ & $\mathbf{1}$ & Total \\
\hline $\mathbf{0}$ & 109 & 29 & 138 \\
$\mathbf{1}$ & 31 & 20 & 51 \\
\hline Total & 140 & 49 & 189 \\
\hline
\end{tabular}

La correlación A7-G8 es $r=0,184(p=.167)$ y no es significativa.

- De los 189 alumnos, solo 20 responden bien al problema de Aritmética y al de Geometría, lo que representa el 10,6 \%.

- Hay 109 alumnos que no responden bien a ninguno de los dos problemas, el $57,7 \%$.

- La correlación entre A7 y G8, es baja y no significativa.

Por género se aplica una t-Student, obteniendo los resultados y la probabilidad que figura en la tabla 7.

Tabla 7. Resultados 2012 por género.

\begin{tabular}{ccccccc}
\hline $\mathbf{2 0 1 2 / 1 3}$ & A7a & A7b & A7 & G8a & G8b & G8 \\
\hline Hombre & 0,605 & 0,447 & 0,447 & 0,789 & 0,289 & 0,211 \\
Mujer & 0,397 & 0,238 & 0,225 & 0,709 & 0,305 & 0,272 \\
$\boldsymbol{p}$-valor & .021 & .010 & .006 & .321 & .856 & .446 \\
\hline
\end{tabular}

- Hay diferencias significativas por género en cada uno de los apartados del problema de Aritmética y en el Total.

- Donde los resultados son más próximos es en el apartado b) de Geometría, con el $p$-valor más alto, próximo a 1.

\section{Resultados 2013 (curso 2013/14)}

La prueba de 2013 fue aplicada a los 71 alumnos de un grupo de de $2 .^{\circ}$ del Grado de Maestro de Primaria al inicio del curso 2013/14, agrupando los resultados en la tabla 8.

Tabla 8. Resultados problemas Aritmética y Geometría 2013.

\begin{tabular}{cccccccc}
\hline 2013/14 & A8a & A8b & A8 & G7a & G7b & G7c & G7 \\
\hline Media & 0,648 & 0,577 & 0,535 & 0,915 & 0,606 & 0,507 & 0,380 \\
D. T. & 0,481 & 0,497 & 0,502 & 0,280 & 0,492 & 0,504 & 0,489 \\
Casos & 71 & 71 & 71 & 71 & 71 & 71 & 71 \\
\hline
\end{tabular}


- El $64,8 \%$ responde bien el apartado a) de la cuestión 8 de Aritmética, el 57,7 \% el apartado b) y el 53,5\% los dos.

- El 91,5\% responde bien el apartado a) de la cuestión 7 de Geometría, el 60,6\% el apartado b), el 50,7\% al c) y el $38 \%$ los tres.

- Los alumnos que responden bien en Aritmética es superior a los que responden bien en Geometría.

El número de alumnos que contestó con puntuación de 0 y 1 aparecen en la tabla 9.

Tabla 9. Número de alumnos por respuesta 2013.

\begin{tabular}{cccc}
\hline $\mathbf{A 8} / \mathbf{G 7}$ & $\mathbf{0}$ & $\mathbf{1}$ & Total \\
\hline $\mathbf{0}$ & 22 & 11 & 33 \\
$\mathbf{1}$ & 22 & 16 & 38 \\
\hline Total & 44 & 27 & 71 \\
\hline
\end{tabular}

La correlación A8-G7 es $r=0,090(p=1,000)$. No existe correlación.

- Solo 16 alumnos responden bien al problema de Aritmética y al de Geometría, que representa el 22,5\%.

- Hay 22 alumnos que no responden bien a ninguno de los dos problemas, el 30,1\%.

- La correlación entre A7 y G8, no existe.

Para ver si hay si hay diferencias significativas por género, al igual que en casos anteriores se aplica la t-Student, obtenido los resultados que aparecen en la tabla 10, sombreando la probabilidad $p<.05$.

Tabla 10. Resultados 2013 por género.

\begin{tabular}{cccccccc}
\hline $\mathbf{2 0 1 3 / 1 4}$ & A8a & A8b & A8 & G7a & G7b & G7c & G7 \\
\hline Hombre & 0,619 & 0,524 & 0,524 & 1,000 & 0,762 & 0,810 & 0,619 \\
Mujer & 0,660 & 0,600 & 0,540 & 0,880 & 0,540 & 0,380 & 0,280 \\
$\boldsymbol{p}$-valor & .746 & .560 & .902 & .100 & .083 & .001 & .007 \\
\hline
\end{tabular}

- Hay diferencias significativas por género en el tercer apartado del problema de Geometría y en el Total.

- Donde los resultados son más próximos es en el apartado a) de Aritmética y en el Total, con el p-valor más alto, próximo a 1. 


\section{Resultados 2014 (curso 2014/15)}

La prueba de 2014, aplicada a 60 alumnos de un grupo de 2. ${ }^{\circ}$ del Grado de Maestro de Primaria al inicio del curso 2014/15, a diferencia de los casos anteriores, contenía dos problemas de Aritmética y dos de Geometría, por lo que se presentan dos tablas (11 y 12), una para Aritmética y otra para Geometría, denominando por A y G los totales.

Tabla 11. Resultados problemas Aritmética y Geometría 2014 (1).

\begin{tabular}{cccccccc}
\hline 2014/15 & A5a & A5b & A5 & A8a & A8b & A8 & A \\
\hline Media & 0,683 & 0,700 & 0,550 & 0,783 & 0,533 & 0,517 & 0,367 \\
D. T. & 0,469 & 0,462 & 0,502 & 0,415 & 0,503 & 0,504 & 0,486 \\
Casos & 60 & 60 & 60 & 60 & 60 & 60 & 60 \\
\hline
\end{tabular}

Tabla 12. Resultados problemas Aritmética y Geometría 2014 (2).

\begin{tabular}{cccccc}
\hline 2014/15 & G9 & G10a & G10b & G10 & G \\
\hline Media & 0,350 & 0,433 & 0,550 & 0,417 & 0,217 \\
D. T. & 0,481 & 0,500 & 0,502 & 0,497 & 0,415 \\
Casos & 60 & 60 & 60 & 60 & 60 \\
\hline
\end{tabular}

- Mientras que el 55,0 \% responde bien al primer problema de Aritmética y el $51,7 \%$ al segundo, solo el $36,7 \%$ de los alumnos responden bien a los dos.

- El primer problema de Geometría lo responde bien el 35,0 \% y el segundo el $41,7 \%$ y los dos el $21,7 \%$.

El número de alumnos que contestó con puntuación de 0 o 1 aparece en la tabla 13.

Tabla 13. Número de alumnos por respuesta 2014.

\begin{tabular}{cccc}
\hline A/G & $\mathbf{0}$ & $\mathbf{1}$ & Total \\
\hline $\mathbf{0}$ & 33 & 5 & 38 \\
$\mathbf{1}$ & 14 & 8 & 22 \\
\hline Total & 47 & 13 & 60 \\
\hline
\end{tabular}

La correlación A-G es $r=0,272(p=.001)$. Es significativa. 
- Solo 8 alumnos responden bien a los problemas de Aritmética y de Geometría, que representa el 13,3\%.

- Hay 33 alumnos que no responden bien a ninguno de los dos problemas, el 55,0\%.

- La correlación entre A y G, es significativa.

Por género, en las tablas 14 y 15 se recogen puntuaciones y probabilidad al aplicar la t-Student para ver si existen diferencias significativas.

Tabla 14. Resultados 2014 por género (1).

\begin{tabular}{cccccccc}
\hline Aritmética & A5a & A5b & A5 & A8a & A8b & A8 & A \\
\hline Hombre & 0,778 & 0,889 & 0,722 & 0,944 & 0,556 & 0,556 & 0,444 \\
Mujer & 0,643 & 0,619 & 0,476 & 0,714 & 0,524 & 0,500 & 0,330 \\
p-valor & .311 & .037 & .082 & .048 & .825 & .699 & .422 \\
\hline
\end{tabular}

Tabla 15. Resultados 2014 por género (2).

\begin{tabular}{cccccc}
\hline Geometría & G9 & G10a & G10b & G10 & G \\
\hline Hombre & 0,444 & 0,611 & 0,778 & 0,610 & 0,333 \\
Mujer & 0,310 & 0,357 & 0,452 & 0,333 & 0,167 \\
p-valor & .324 & .071 & .020 & .046 & .156 \\
\hline
\end{tabular}

- Hay diferencias significativas por género en dos de los apartados de los problemas de Aritmética y en uno de los problemas de Geometría.

\section{Resultados de 2011/12 a 2014/15}

En la tabla 16 viene un resumen de los resultados por curso y género en Aritmética y Geometría $(\mathrm{AH}=$ Aritmética Hombres, $\mathrm{AM}=$ Aritmética Mujeres, $\mathrm{GH}=$ Geometría Hombres, GM = Geometría Mujeres, $\mathrm{A}=$ Aritmética y $\mathrm{G}=$ Geometría), señalando en sombreado los más altos y en negrita los más bajos por columna.

Tabla 16. Resumen de Álgebra y Geometría por género.

\begin{tabular}{ccccccc}
\hline & AH & AM & GH & GM & A & G \\
\hline $\mathbf{2 0 1 1 / 1 2}$ & 86,4 & 81,2 & 9,1 & $\mathbf{8 , 3}$ & 82,9 & $\mathbf{8 , 6}$ \\
$\mathbf{2 0 1 2 / 1 3}$ & 44,7 & $\mathbf{2 2 , 5}$ & 21,1 & 27,2 & $\mathbf{2 7 , 0}$ & 26,5 \\
$\mathbf{2 0 1 3 / 1 4}$ & 52,4 & 54,0 & $\mathbf{6 1 , 9}$ & 28,0 & 53,5 & 38,0 \\
$\mathbf{2 0 1 4 / 1 5}$ & $\mathbf{4 4 , 4}$ & 33,0 & 33,3 & 16,7 & 36,7 & 21,7 \\
\hline
\end{tabular}


- En los cuatro cursos los resultados en Aritmética son mejores que los de Geometría.

- El problema de Geometría en el curso 2011/12 es el que obtiene peores resultados.

- En ningún curso los problemas de Geometría han sido resueltos por más del 38,0 \% de los alumnos.

- Solo en el curso 2012/13 los resultados en Geometría y Aritmética son similares.

- Comparando por género, de los ocho resultados, en seis son mejores los hombres.

\section{Discusión}

Los más de 50000 alumnos de $6 .^{\circ}$ de Primaria de la Comunidad de Madrid (6PCM), en los años que han sido publicados los resultados, obtuvieron en los problemas de la muestra (CAM, 2014) unos porcentajes de aciertos, que se comparan con los del estudio con futuros maestros (FM). Se presentan en las tablas 17, 18 y 19.

Tabla 17. Comparativa con resultados CDI 2012 de 6. ${ }^{\circ}$ Primaria.

\begin{tabular}{ccccc}
\hline $\mathbf{2 0 1 2}$ & A7a & A7b & G8a & G8b \\
\hline 6PCM & 29,2 & 14,5 & 51,7 & 26,6 \\
FM & 43,9 & 28,0 & 72,5 & 30,2 \\
\hline
\end{tabular}

Tabla 18. Comparativa con resultados CDI 2013 de 6. ${ }^{\circ}$ Primaria.

\begin{tabular}{cccccc}
\hline $\mathbf{2 0 1 3}$ & A8a & A8b & G7a & G7b & G7c \\
\hline 6PCM & 63,7 & 53,7 & 77,4 & 32,3 & 53,9 \\
FM & 64,8 & 57,7 & 91,5 & 60,0 & 50,7 \\
\hline
\end{tabular}

Tabla 19. Comparativa con resultados CDI 2014 de 6. ${ }^{\circ}$ Primaria.

\begin{tabular}{cccccccc}
\hline $\mathbf{2 0 1 4}$ & A5a & A5b & A8a & A8b & G9 & G10a & G10b \\
\hline 6PCM & 50,8 & 71,6 & 72,1 & 67,3 & 32,9 & 37,7 & 54,7 \\
FM & 68,3 & 70,0 & 78,3 & 53,3 & 35,0 & 43,3 & 55,0 \\
\hline
\end{tabular}

- En el curso 2012/13 los porcentajes de aciertos de los futuros maestros son superiores a los de los alumnos de $6 .^{\circ}$ de Primaria.

- En el curso 2013/14 estos resultados están más próximos. 
- En el curso 2014/15 en dos casos obtienen mejores resultados los alumnos de $6 .^{\circ}$ de Primaria.

De los diez problemas analizados, cinco de Aritmética y cinco de Geometría, los porcentajes de resultados correctos de los futuros maestros se resume en la tabla 20, en donde 20141 y 20142 representan los resultados del primero y segundo problema de 2014, respectivamente:

Tabla 20. Resumen final de resultados.

\begin{tabular}{lccccc}
\hline & $\mathbf{2 0 1 1}$ & $\mathbf{2 0 1 2}$ & $\mathbf{2 0 1 3}$ & $\mathbf{2 0 1 4 1}$ & $\mathbf{2 0 1 4 2}$ \\
\hline Aritmética & 82,9 & 27,8 & 57,7 & 55,0 & 51,7 \\
Geometría & 8,6 & 26,5 & 38,0 & 35,0 & 41,7 \\
\hline
\end{tabular}

En cuanto a los bloques de Aritmética y Geometría en todos los cursos se tiene mejor resultado en Aritmética que en Geometría, siendo en Aritmética en dos cursos un porcentaje de aciertos superior al $50 \%$, pero en Geometría el porcentaje más alto de aciertos es del $38 \%$.

Por género, no se encuentran diferencias significativas en el curso 2011/12 ni en Aritmética ni en Geometría; en el curso 2012/13 en el problema de Aritmética hay diferencias significativas ( $p=.006$ ), en 2013/14 hay diferencias significativas $(p=.007)$ en el problema de Geometría y $2014 / 15$ hay diferencias significativas $(p=.046)$ en todos los casos siempre favorables a hombres frente a mujeres. Solo en el curso 2014/2015 la correlación entre resultados de Aritmética y Geometría es significativa $(r=0,272$ y $p=.001)$.

En Nortes (2011) al aplicar la prueba CDI (2009) a alumnos de $2 .^{\circ}$ de la Diplomatura de Maestro de E. Primaria, E. Física y Lenguas extranjeras, en una muestra de 235 alumnos, de los que 69 correspondían a $2 .^{\circ}$ de la Diplomatura de E. Primaria, se propusieron cinco problemas, cuatro de Aritmética y uno de Geometría, de enunciado: "Se quiere cubrir con baldosas cuadradas de $20 \mathrm{~cm}$ de lado el suelo de una habitación que mide 3 metros de lado. a) Calcula, en cm, el perímetro de la habitación y b) ¿Cuántas baldosas se necesitan" que fue contestado con acierto cada apartado por el 50,9 \% y $18,7 \%$, respectivamente, lo que corrobora la baja competencia matemática de los futuros maestros, ya que de los cuatro problemas de Aritmética analizados, con dos cuestiones cada uno, tan solo el apartado de expresar en unidades de tiempo (h, min, s) no superó el $50 \%$ de aprobados (30,5\%), lo que se reafirma en este 
estudio de que los problemas de Aritmética se resuelven mejor que los de Geometría.

En el estudio realizado por Liñán, Barrera e Infante (2014) con el planteamiento de un problema con división de fracciones y ante el resultado del mismo, se preguntan cómo pueden afectar estas deficiencias al desarrollo de las demás componentes del conocimiento especializado del profesor de matemáticas.

Si revisamos los estándares de aprendizaje evaluables en el RD 126/2014 referentes a la Enseñanza Primaria para ver si un alumno resuelve problemas que impliquen dominio de los contenidos trabajados, utilizando estrategias heurísticas de razonamiento, creando conjeturas, construyendo, argumentando... tendríamos que decir que tanto en Aritmética como en Geometría, los futuros maestros no los cumplen y Liñán y Contreras (2013) plantean si es competencia de los centros de formación inicial de maestros rellenar las lagunas de conocimientos con los que llegan los estudiantes para maestro.

\section{Conclusiones}

A la vista de los resultados podemos afirmar con palabras de Salinas (2007) que a lo largo de nuestra práctica docente, como formadores de maestros, venimos constatando en nuestros alumnos lagunas de conocimientos, errores conceptuales en contenidos matemáticos que deberían haberse adquirido en los primeros años de la Enseñanza Primaria.

Que en ninguno de los cuatro cursos los problemas de Geometría hayan tenido más de un $38 \%$ de aciertos y que los problemas de Aritmética solo dos años superaron el $50 \%$ de aciertos nos reafirma en la cita anterior, lo que nos llevaría a compartir lo dicho por Barrera, Infante y Liñán (2013) al afirmar que diversos trabajos muestran errores y carencias de determinados conceptos, dificultades en la aplicación de los mismos y cuando las respuestas son correctas hay estudiantes para maestro que no saben justificarlas adecuadamente. Lo que nos lleva a plantear la pregunta tan repetida ¿en qué falla el sistema educativo? Porque los alumnos de $6 .^{\circ}$ de Primaria de la Comunidad de Madrid en algunos de los problemas planteados obtienen mayor porcentaje de aciertos en la resolución de problemas que los futuros maestros y en otros las diferencias son muy exiguas, como en el caso de la prueba de 2014. 
Cabría decir, para finalizar, y con vistas a completar esta investigación, que los resultados en Aritmética, -pese a que el $60 \%$ de la muestra de Ruiz de Gauna et al. (2013) no la reconocían como bloque- son mejores que los obtenidos en Geometría, aún siendo ambos bajos, lo que nos lleva a la conclusión de que hay que mejorar la formación de los maestros, siendo necesario establecer unas bases comunes y este conocimiento matemático imprescindible para iniciar el Grado de Maestro de Primaria no debería hacerse como dicen Castro et al. (2014) al margen de los departamentos de Didáctica de la Matemática.

En próximos estudios se seguirá investigando la formación inicial de los futuros maestros, porque como dicen Cantón, Cañón, y Arias (2013) "La decisiva influencia de los profesores en la vida de los alumnos hace que el profesorado se convierta en objeto de estudio en multitud de investigaciones, trabajos y reflexiones" (p. 46).

\section{Referencias bibliográficas}

Barrera, V., Infante, J. M. y Liñán, M. M. (2013). Conocimiento matemático común en geometría de los estudiantes para maestro: una propuesta de innovación. Escuela Abierta, 16, 11-33.

Cantón, I., Cañón, R. y Arias, A.R. (2013). La formación universitaria de los maestros de Educación Primaria. Revista Interuniversitaria de Formación del Profesorado, 76 (27, 1), 45-63.

CAM (2014). Pruebas de Conocimientos y Destrezas Indispensables (CDI) de $6 .^{\circ}$ de Educación Primaria. www.madrid.org.

Castro, A., Mengual, E., Prat, M. Albarracín, L. y Gorgorió, N. (2014). Conocimiento matemático fundamental para e Grado de Educación Primaria: inicio de una línea de investigación. En M.T. González, M. Codes, D. Arnau y T. Ortega (Eds.), Investigación en Educación Matemática XVIII (pp. 227-236). Salamanca: SEIEM.

Castro, A., Gorgorió, N. y Prat, N. (2015). Conocimiento matemático fundamental en el Grado de Educación Primaria: sistema de numeración decimal y valor posicional. En C. Fernández, M. Molina y N. Planas (eds.), Investigación en Educación Matemática XIX (pp. 221-228). Alicante: SEIEM.

Recuperado de Decreto . $^{\circ}$ 198/2014, de 5 de septiembre, por el que se establece el currículo de la Educación Primaria en la Comunidad Autónoma de la Región de Murcia. BORM n. ${ }^{\circ} 206$ de 6/07/2014.

Escudero-Domínguez, A. y Carrillo, J. (2014). Conocimientos matemáticos sobre cuadriláteros en estudiantes para maestro. En M.T. González, M. Codes, D. Arnau y T. Ortega (Eds.), Investigación en Educación Matemática XVIII (pp. 267-276). Salamanca; SEIEM. 
García, A., Buforn, A. y Torregrosa, G. (2014). Un módulo de enseñanza centrado en desarrollar el razonamiento configural: características desde una perspectiva cognitiva. En M.T Tortosa, J.D. Álvarez y N. Pellín (coord.), XII Jornadas de Redes de Investigación en Docencia Universitaria. Alicante: Universidad de Alicante.

Liñán, M.M. y Contreras, L.C. (2013). Debilidades y fortalezas en el conocimiento de los temas matemáticos en Geometría de los estudiantes para maestro. En A. Berciano, A. Estepa y N. Climent (Eds.), Investigación en Educación Matemática XVII (pp. 337343). Santander: SEIEM.

Liñán, M.M., Barrera, V. e Infante, J.L. (2014). Conocimiento especializado de los estudiantes para maestro: la resolución de un problema con división de fracciones. Escuela Abierta, 17, 41-63.

Montes, M.A., Contreras, L.C., Liñán, M.M., Muñoz-Catalán, M.C., Climent, N. y CarriIlo, J. (2015). Conocimiento de aritmética de futuros maestros. Debilidades y fortalezas. Revista de Educación, 367, 36-62.

NCTM (2000). Principios y Estándares para la Educación Matemática. Edición en español 2003. Granada: Sociedad Andaluza de Educación Matemática Thales.

Orden ECl/3858/2007, de 27 de diciembre, por la que se establecen los requisitos para la verificación de los títulos universitarios oficiales que habiliten para el ejercicio de las profesiones de Profesor de Educación Secundaria Obligatoria y Bachillerato, Formación Profesional y Enseñanzas de Idiomas. BOE, n. 312 de 29/12/2007.

Real Decreto 126/2014, de 28 de febrero, por el que se establece el currículo básico de la Educación Primaria. BOE, n. ${ }^{\circ} 52$ de 1/03/2014.

Nortes Martínez-Artero, R. (2011). Pruebas de conocimientos en Matemáticas de $6^{\circ}$ de Educación Primaria: una aplicación a futuros maestros. En A. de Pro, Investigación e Innovación en Educación Infantil y Educación Primaria III (pp. 527-538). Mucia: Universidad de Murcia.

Ruiz de Gauna, J., García, J. y Sarasua, J. (2013). Perspectiva de los alumnos de Grado de Educación Primaria sobre las Matemáticas y su enseñanza. Números, 82, 5-15.

Salinas, M.J. (2007). Errores sobre el sistema de numeración decimal en estudiantes de magisterio. En M. Camacho, P. Flores y P. Bolea (Eds.), Investigación en Educación Matemática XI, (pp. 381-390). La Laguna: SEIEM.

Torregrosa, G., Quesada, H. y Penalva, M. C. (2010). Razonamiento configural como coordinación de procesos de visualización. Enseñanza de las Ciencias, 28(3), 327340 . 
